UUITP-03-08

\title{
Dimensional Regularization and Dimensional Reduction in the Light Cone
}

\author{
J.Qiu \\ Department of Physics and Astronomy \\ Uppsala University \\ Sweden \\ (November 2, 2018)
}

\begin{abstract}
We calculate all the 2 to 2 scattering process in Yang-Mills theory in the Light Cone gauge, with the dimensional regulator as the UV regulator. The IR is regulated with a cutoff in $q^{+}$. It supplements our earlier work, where a Lorentz non-covariant regulator was used and the final results bear some problems in gauge fixing. Supersymmetry relations among various amplitudes are checked using the light cone superfields.
\end{abstract}




\section{Introduction}

The recent years saw a fast development in the techniques of perturbative computation of amplitudes in Yang-Mills theory. Among these are string based computation [1], unitarity based techniques [2], and MHV techniques [3] inspired by the twistor string formalism. Thanks to these new developments, a large class of amplitudes can now be computed. In particular, for the $\mathrm{N}=4$ supersymmetric Yang-Mills (MSYM) theory, certain amplitudes have been calculated to quite high loop orders [4]. And an iteration relation to all orders was postulated by Bern, Dixon and Smirnov (BDS) [5]. This iteration relation became all the more interesting in the light of [6], where the gluon scattering amplitude is calculated at strong coupling using $\mathrm{AdS} / \mathrm{CFT}^{1}$.

The present article is dedicated to the computation of the scattering amplitudes in Yang-Mills theory in the light cone gauge. The purpose of this work is, however, not to bring coals to Newcastle. Admittedly, our ability to perform computation in the light cone gauge is severely marginalized when compared to the others using advanced and streamlined techniques. But there are plenty of occasions when a 4D computation is desirable, or when we would like each field in the lagrangian to correspond to one particle exactly. The first of our series of computations was necessitated by the need to study the renormalization of YM theory represented on the light cone world-sheet (see [8] for an introduction), in the selfsame paper we found some novel (local world sheet) counter terms required to restore gauge invariance. Recently, when attention was paid to the search for the Lagrangian origin of MHV rules [9], the ++- (non-MHV) vertex present in the light cone lagrangian was absorbed into the kinetic term through a change of variable, and one of the counter terms found in [8] was interpreted as part of the Jacobian associated to the changing of variable [10]. In addition to the novel counter terms, we also found a nice infrared subtraction scheme tailored for the light cone in [11]. This nice separation of an amplitude into a hard part(infrared free) and a soft part was in fact a surprise to us, for neither party was expected to be Lorentz covariant on its own with our simple infrared subtraction.

In our past works $[8,11-13]$, we have computed the scattering amplitudes in the light cone gauge with a $\delta$-regulator (an exponential damping in the transverse direction) to tame the UV. This regulator has some peculiar features in terms of breaking the gauge invariance. Naturally all the breaking of gauge invariance can be restored with proper counter terms, but the little freedom in choosing the counter terms leaves the amplitudes defined only up to a constant multiple of a tree. Thus only in special cases such as MSYM, where all counter terms vanish, can we check the supersymmetric relations among various amplitudes. So we turn to using a dimensional regulator specially modified for the light cone, first and foremost as a check for the calculation done in our past work, and yet we also expect it to have some spin off's. The main feature of the dimensional regulator used in this article is that it only regulates UV divergence leaving the IR regulated by setting the + component of the loop momenta away from zero as was done in [11]. This regularization scheme turned out just as efficient, in terms that the final result is automatically gauge invariant, and in the case of MSYM, bears no reference to the dimension parameter $\epsilon$.

In this article, we use both the dimensional regularization or 't Hooft-Veltman scheme [14], and dimensional reduction [15]. To save space we shall refer to the former as HV scheme and the latter as DR scheme. HV scheme is known to break supersymmetry $[15,16]$, and does not go well with the helicity based calculation. These are merely two different facets of the same problem. In $4 \mathrm{D}$, the orthochronous component of $S O(3,1)$ has a double covering $S L(2, C)$, which enables us to express a polarization vector as a product of two spinors of the form $\mid \lambda]\langle k|$, and by picking the reference spinor $\mid \lambda]$ wisely, we spare ourself the trouble of computing a large class of diagrams. Sometimes this makes all the difference between being able or not able to perform certain computations. One cannot use the same strategy in a different dimension, and a related problem is whether or not the matrices of the form $\gamma^{\left[\mu_{1}\right.} \gamma^{\mu_{2}} \ldots \gamma^{\left.\mu_{i}\right]}$ form a complete basis as they do in 4D. This would tell on the validity of Fierz identity, and thereby the preservation of supersymmetry [17]. With DR scheme, all of the above problems are absent, the result preserves gauge symmetry and supersymmetry. Our modified DR scheme produce results that conform to this common lore.

This article is organized as follows, sec.2 contains the strategy of our dimensional regulation schemes

\footnotetext{
${ }^{1}$ a review paper [7] become available during the preparation of the paper
} 
and some examples. Computational results are listed in sec.3. Although the loop calculations are done in the component fields, we use light cone superfield formalism to discuss the supersymmetric relations among various amplitudes in sec.4. Sec.5 contains the discussion of the infrared parts regulated in the light cone fashion, which is the major novel feature of our regulation scheme. In the appendix, some intermediate computational results are listed and the light cone super field is also summarized there.

\section{Dimensional Regularization in the Light Cone}

The light cone singles out the $t$ and $z$ direction of the space time, leaving the $x$ and $y$ direction as the transverse dimensions. So a natural choice of HV scheme suited for the light cone is to set the number of transverse direction to be $D=2-2 \epsilon$. Now it is crucial that our dimensional regulator does not touch upon the IR part, so any possible difference in the result between using a UV $\delta$-regulator and dimensional regulator will only come from the superficially UV divergent part ${ }^{2}$.

With this observation, we need only focus on the potentially divergent part of an amplitude, and study the possible difference resulting in applying either $\delta$ or $H V$ regulator.

As stated in the introduction, a helicity based calculation is much preferred, for it grants us the ability to apply some on-shell identities of the amplitudes. We can apply this method equally well in the light cone, with the polarization vectors defined as

$$
\epsilon_{\vee}^{\mu}=\frac{1}{\sqrt{2}}\left(\frac{k^{1}-i k^{2}}{k^{0}+k^{3}}, 1,-i,-\frac{k^{1}-i k^{2}}{k^{0}+k^{3}}\right)=\frac{1}{\sqrt{2}}\left(\frac{k^{\wedge}}{k^{+}}, 1,-i,-\frac{k^{\wedge}}{k^{+}}\right) ; \epsilon_{\wedge}=\epsilon_{\vee}^{*}
$$

where the subscript $\wedge \vee$ correspond to the positive and negative helicity. With the HV scheme, we have a total of $D=2-2 \epsilon$ polarizations for a gluon. Then it become awkward to use the $\wedge \vee$ basis. In particular, we have problems interpreting terms like $\delta_{i}^{\wedge}$, where $i$ ranges from $2-2 \epsilon$ to 2 . Especially any error we make might be amplified by a factor of $1 / \epsilon$ and become non-negligible.

To avoid the ambiguities, we need to treat all the $2-2 \epsilon$ transverse direction as equal by adopting a cartesian basis. In the cartesian basis, the polarization vector relevant for the light cone become

$$
\epsilon_{i}^{\mu}=\left(\frac{1}{\sqrt{2}} \frac{k^{i}}{k^{+}}, \ldots, \delta_{i}^{\mu}, \ldots,-\frac{1}{\sqrt{2}} \frac{k^{i}}{k^{+}}\right)
$$

where the subscript $i$ is a label that labels the ' $i{ }^{t h}$ ' polarity of a gluon. The - component of $\epsilon_{i}^{\mu}$ is thus $k^{i} / k^{+}$, the + component is of course zero corresponding to the light cone gauge fixing. While for the transverse components, only the $i^{t h}$ take a 1 with all the others 0 . In the appendix as well as in [12], we give some properties of this polarization vector. With this setup, the transverse dimension are treated on equal footing, instead of being further divided into a 2 and $D-2$ part, which will make the calculation rather untidy. As for the amplitudes involving fermions, we face a larger problem of manipulating the gamma matrices in a non-integer dimension, but so long as we adopt the cartesian basis and stick to Dirac fermions (staying away from $\gamma^{5}$ ), there is no conceptual difficulty in the interpretation (as we shall see later).

We now give an example of this procedure. Consider a generic box diagram, after the usual steps of exponentiating the denominators, shifting the momenta etc, we can write down the divergent part of this box integrand.

$$
B_{H V}=\mu^{2 \epsilon} \int \frac{d^{D} q_{\perp}}{(2 \pi)^{D}} \int_{0}^{\infty} \frac{T^{3} d T}{2 T} \int_{0<x_{i}<1} d^{4} x_{i} \delta\left(\sum x_{i}-1\right) q_{\perp}^{4} \exp \left(-i T q_{\perp}^{2}+i T H\right) B(2+D)
$$

where $B(2+D)$ is an object that encapsules all the $D+2$ dimensional tensor algebra or gamma matrix algebra and $H$ is just some function of the Schwinger parameters that need not concern us now. The same

\footnotetext{
${ }^{2}$ Note the separation of an amplitude into UV divergent part and finite part is highly gauge dependent, and even procedure dependent, a point to bear in mind
} 
integrand written with a $\delta$-regulator is

$$
B_{\delta}=\int \frac{d^{2} q_{\perp}}{(2 \pi)^{2}} \int_{0}^{\infty} \frac{T^{3} d T}{2 T} d^{4} x_{i} \delta\left(\sum x_{i}-1\right) q_{\perp}^{4} \exp \left(-i(T-i \delta) q_{\perp}^{2}+i T H\right) B(4)
$$

The evaluation is straight forward, we get for HV scheme

$$
B_{H V}=\int d^{4} x_{i} \delta\left(\sum x_{i}-1\right) \frac{i}{8 \pi^{2}} \frac{D(2+D)}{8} B(2+D)\left[\frac{1}{\epsilon}-\gamma\right]\left[1-\epsilon \log \frac{H}{\mu^{2}}+\epsilon \log 4 \pi\right]
$$

where $\epsilon$ is $(2-D) / 2$, and we can also redefine $\mu$ to absorb $\log 4 \pi$. Using $\delta$-regulator, we have

$$
B_{\delta}=\int d^{4} x_{i} \delta\left(\sum x_{i}-1\right) \frac{i}{8 \pi^{2}} B(4)\left[-\log \delta H e^{\gamma}-\frac{3}{2}\right]
$$

We are only interested in the difference between these two schemes

$$
B_{H V}-B_{\delta}=\int d^{4} x_{i} \delta\left(\sum x_{i}-1\right) \frac{i}{8 \pi^{2}}\left[\left(\frac{1}{\epsilon}+\log \mu^{2}\right) B(2+D)+\log (\delta) B(4)\right]+\mathcal{O}(\epsilon)
$$

Clearly $1 / \epsilon+\log \mu^{2}$ is to be identified with $-\log \delta$, hence

$$
B_{H V}-B_{\delta}=\frac{i}{8 \pi^{2}} \lim _{\epsilon \rightarrow 0} \frac{1}{\epsilon}[B(2+D)-B(4)]
$$

Note the dependence of $B(2+D)$ upon $D$ is two fold, one explicit dependence for which we can differentiate, take limits etc; the other intrinsic dependence that does not allow for such manipulations. So some care is needed in dealing with Eq.8, especially when gamma matrices are involved (for example a term $\sum \gamma^{i} \gamma^{i} / \epsilon$, where the summation is from $2-2 \epsilon$ to 2 , is hard to give meaning to).

For self-mass and vertex corrections, we may try to compute the wave function or charge renormalization (whose $D$ dependence is obviously differentiable) to circumvent the possible ambiguity of Eq.8. For the scattering amplitude, we will compute the superficially divergent part, the terms that has $1 / \epsilon$ poles will be kept to the very end, where they have to fall together to be proportional to a tree. Note this tree is defined in $2+D$ dimension and this is done unambiguously only by using cartesian basis. In the same manner for the $\delta$-regulator, terms multiplying $\log \delta$ must also be proportional to a tree (defined in 4 dimension). At this stage we will identify $\left(1 / \epsilon+\log \mu^{2}\right) \cdot \operatorname{tree}(2+D)$ with $-\log \delta \cdot \operatorname{tree}(4)$. After this identification, whatever is left will be finite but will not in general be proportional to a tree, in fact, they will have to compensate for the breaking of gauge invariance due to the $\delta$-regulator (the example is given in sec.2.4).

\subsection{Box Diagrams}

We shall apply the above procedure to the box diagrams as follows: denote by $B^{\mu \nu \rho \sigma}$ a generic four gluon box diagram, with external indices unspecified. Let

$$
B\left(g^{i}, g^{j}, g^{k}, g^{l}\right) \equiv\left(-\epsilon_{i \mu}\right)\left(-\epsilon_{j \nu}\right)\left(-\epsilon_{k \rho}\right)\left(-\epsilon_{l \sigma}\right) B^{\mu \nu \rho \sigma}
$$

be a box where the four external gluons are physically polarized. We emphasize that the indices $i, j \ldots$ are not space time indices, but rather labels that label the different polarities. Since a box diagram in the light cone gauge is logarithmic divergent, the indices $i, j \ldots$ will only be carried by Kronecker delta symbols. We get (the argument behind the semicolon:'g','s' or 'q' means that gluon, scalar or fermion circulates the loop)

$$
\begin{aligned}
& B\left(g^{i}, g^{j}, g^{k}, g^{l} ; g\right)=\frac{2}{2-D}\left\{\left[\delta^{i l} \delta^{j k}+\delta^{i j} \delta^{k l}\right]\left[\frac{8}{3(2+D)}-\frac{2}{D}+\frac{1}{2}\right]+\delta^{i k} \delta^{j l} \frac{8}{3(2+D)}+\text { Art. Div. }\right\} \\
& B\left(g^{i}, g^{j}, g^{k}, g^{l} ; s\right)=\frac{2}{2-D}\left[\delta^{i l} \delta^{j k}+\delta^{i j} \delta^{k l}+\delta^{i k} \delta^{j l}\right] \frac{8}{3 D(2+D)} \\
& B\left(g^{i}, g^{j}, g^{k}, g^{l} ; q\right)=\frac{2}{2-D}\left\{\left[\delta^{i l} \delta^{j k}+\delta^{i j} \delta^{k l}\right] \frac{-4\left(3 D^{2}-4\right)}{3 D(2+D)}+\delta^{i k} \delta^{j l} \frac{4\left(3 D^{2}-8+6 D\right)}{3 D(2+D)}\right\}
\end{aligned}
$$


where we omit a factor $i g^{4} N_{c} /\left(8 \pi^{2}\right) \operatorname{Tr} t^{a} t^{b} t^{c} t^{d}$. The results are presented so that we can get the $1 / \epsilon$ pole by taking $D$ to 2 , or the difference $\mathrm{HV}$ versus $\delta$ by extracting the finite part as $D \rightarrow 2$. Note that the difference in the tensor structure among the above three is expected. $B\left(g^{i}, g^{j}, g^{k}, g^{l} ; q\right)$ must be finite when summed over all possible cyclic orderings, corresponding to the finiteness of the four photon box in QED. $B\left(g^{i}, g^{j}, g^{k}, g^{l} ; g\right)$ has the same property, which simply says photons do not interact with photons in the absence of the electrons (called $U(1)$-decoupling).

'Art. Div' stands for 'gauge artificial divergence' and is poles in $q^{+}$. They are too cumbersome to list and they in general will be $D$ dependent. All the final results must go through the non-trivial test that the gauge artificial divergences cancel up to $\mathcal{O}(\epsilon)$.

Another example is $B\left(g^{i}, g^{j}, q, \bar{q}\right)$, a box with two fermion legs and two gluon legs. This diagram would have been finite by naive power counting, however, in the light cone set up, we have terms like $q^{i} / q^{+}$that will be missed by the naive power counting (we do not adopt the Mandelstam prescription, so a Wick rotation is not valid), but actually contributing to the UV divergence. But we can still see the trace of naive power counting from the fact that its $1 / \epsilon$ pole contribution consists solely of gauge artificial divergences, i.e. of order $\mathcal{O}\left(1 / q^{+}\right)$, conforming to the result of naive power counting.

\subsection{Self-mass Diagrams}

The bosonic self-mass diagrams are similar, and the results using dimensional regularization must be gauge covariant(transverse). We first list the $\delta$-regulator results (with the mass renormalization term and a factor of $i g^{2} N_{c} /\left(8 \pi^{2}\right) \operatorname{Tr}\left[t^{a} t^{b}\right]$ omitted $)$

$$
\begin{array}{lll}
\Pi\left(g^{i}, g^{j} ; s\right)=\delta^{i j} p^{2}\left[\frac{5}{18}-\frac{1}{6} \log p^{2} \delta e^{\gamma}\right] & \Pi(+,+; s)=p^{+2}\left[\frac{4}{9}-\frac{1}{6} \log p^{2} \delta e^{\gamma}\right] \\
\Pi\left(g^{i}, g^{j} ; q\right)=\delta^{i j} p^{2}\left[\frac{26}{9}-\frac{4}{3} \log p^{2} \delta e^{\gamma}\right] & \Pi(+,+; q)=p^{+2}\left[\frac{20}{9}-\frac{4}{3} \log p^{2} \delta e^{\gamma}\right] \\
\Pi\left(g^{i}, g^{j}, g\right)=\delta^{i j} p^{2}\left[-\frac{67}{9}+\frac{11}{3} \log p^{2} \delta e^{\gamma}+\int_{0}^{p^{+}} d q^{+}\left[\frac{-2}{p^{+}-q^{+}}+\frac{-2}{q^{+}}\right] \log \frac{\left(p^{+}-q^{+}\right) q^{+} p^{2} \delta e^{\gamma}}{p^{+2}}\right] \\
\Pi(+,+, g)=p^{+2}\left[\frac{8}{9}-\frac{1}{3} \log p^{2} \delta e^{\gamma}\right]
\end{array}
$$

where $g^{i}$ tells the polarity of the gluon, while $\Pi(+,+)$ describes the propagation of $A^{-}$. These results are not Lorentz covariant, as can be seen from the fact that the numbers in $\Pi\left(g^{i}, g^{j}\right)$ do not match those in $\Pi(+,+)$. The procedure in the previous section must produce a compensation to these mismatches. Indeed, when we evaluate the superficially divergent parts of self-mass diagrams in $2+D$ dimension

$$
\begin{array}{rlrl}
\Pi\left(g^{i}, g^{j} ; g\right) & =\frac{2}{2-D} \delta^{i j} p^{2}\left(-\frac{11}{3}\right) & \Pi(+,+; g)=\frac{2}{2-D} \frac{D}{6} p^{+2} \\
\Pi\left(g^{i}, g^{j} ; s\right)=\frac{2}{2-D} \delta^{i j} p^{2}\left(\frac{1}{3 D}\right) & \Pi(+,+; s)=\frac{2}{2-D} \frac{1}{6} p^{+2} \\
\Pi\left(g^{i}, g^{j} ; q\right)=\frac{2}{2-D} \delta^{i j} p^{2}\left(-\frac{4}{3 D}+2\right) & \Pi(+,+; q)=\frac{2}{2-D} \frac{4}{3} p^{+2}
\end{array}
$$

One can extract the finite terms as $D \rightarrow 2$ according to Eq.8 and combine them to Eq.11, and we gladly see that the Lorentz covariance is restored in $\Pi(g, g ; s)$ and $\Pi(g, g ; q)$.

$$
\Pi(g, g ; q)=-\left(p^{2} g^{\mu \nu}-p^{\mu} p^{\nu}\right)\left[\frac{20}{9}-\frac{4}{3} \log p^{2} \delta e^{\gamma}\right] \quad \Pi(g, g ; s)=-\left(p^{2} g^{\mu \nu}-p^{\mu} p^{\nu}\right)\left[\frac{4}{9}-\frac{1}{6} \log p^{2} \delta e^{\gamma}\right]
$$

$\Pi(g, g ; g)$ contains the infrared and collinear divergence (the integral term in the parenthesis), so the Lorentz covariance is not explicit. 
Next we look at the fermion self-mass diagram. If we can still say that the $D$ dependence in the previous results is explicit so long as we keep the polarization index $i$ between 1 and $D$, we now encounter an intrinsic $D$ dependence through the gamma matrices. So we turn instead to computing the wave function renormalization $Z_{2}$. The superficially divergent terms are calculated to be

$$
\begin{aligned}
\Pi(q, \bar{q}) & =\frac{2}{2-D}\left\{-\frac{D}{2}\left[p_{\perp}^{i} \gamma^{i}-\frac{p_{\perp}^{2}}{2 p^{+}} \gamma^{+}-p^{+} \gamma^{-}\right]+\frac{(D-8) p^{2}}{4 p^{+}} \gamma^{+}\right\} \\
& =\frac{2}{2-D}\left\{\frac{D}{2} \not p_{\perp}+\frac{(D-8) p^{2}}{4 p^{+}} \gamma^{+}\right\}
\end{aligned}
$$

Here we find it handy to introduce the notation of $\not p_{\perp}$, it is an off-shell extrapolation of $\not p$ and obeys $\not p_{\perp} \cdot \not p_{\perp}=0$. The result above is not proportional to $\not p$, as is necessary to compute $Z_{2}$. When we try to embed it into a larger diagram by connecting two propagators to it

$$
\begin{aligned}
& \frac{i \not p}{p^{2}} \frac{2}{2-D}\left\{\frac{2}{D} \not p_{\perp}+\frac{(D-8) p^{2}}{4 p^{+}} \gamma^{+}\right\} \frac{i \not p}{p^{2}} \\
= & \frac{2}{2-D} \frac{i}{p^{2}}\left[\not p_{\perp}+\frac{p^{2}}{2 p^{+}} \gamma^{+}\right]\left\{\frac{D}{2} \not p_{\perp}+\frac{(D-8) p^{2}}{4 p^{+}} \gamma^{+}\right\}\left[\not p_{\perp}+\frac{p^{2}}{2 p^{+}} \gamma^{+}\right] \frac{i}{p^{2}} \\
= & \frac{2}{2-D} \frac{i}{p^{2}}\left[\frac{D}{2} \frac{p^{4}}{2 p^{+}} \gamma^{+}+\frac{(D-8) p^{2}}{2} \not p_{\perp}\right] \frac{i}{p^{2}}=\frac{2}{2-D} \frac{i}{p^{2}}\left[\frac{p^{4}}{2 p^{+}} \gamma^{+}+\frac{(D-8) p^{2}}{2} \not p\right] \frac{i}{p^{2}}
\end{aligned}
$$

we must discard the first term as it contains too many powers of $p^{2}$ rendering the diagram 1PIR. With this observation we can simply take $(D-8) / \epsilon$ as the correction to $Z_{2}$, whose $D$ dependence is differentiable.

\subsection{Triangle Diagrams}

The vertex correction evaluated with a $\delta$-regulator also shows non-gauge-covariance. For example we observe a mismatch between $\Gamma\left(g^{i}, g^{j},+\right)$ and $\Gamma\left(g^{i}, g^{j}, g^{k}\right)$, i.e. a mismatch when one gluon polarization is changed from $i$ to - . We omit the factor $i g^{3} N_{c} /\left(8 \pi^{2}\right) \operatorname{Tr}\left[t^{a} t^{b} t^{c}\right]$

$$
\begin{aligned}
\Gamma\left(g^{i}, g^{j},+; s\right) & =-\left(p_{1}^{+}-p_{2}^{+}\right) \delta^{i j}\left[\frac{1}{6} \log p_{o}^{2} \delta e^{\gamma}-\frac{5}{\underline{18}}\right] \\
\Gamma(g-, g-, g+; s) & =\frac{-2 p_{3}^{+}}{p_{1}^{+} p_{2}^{+}} K_{2,1}^{\wedge}\left[\frac{1}{6} \log p_{o}^{2} \delta e^{\gamma}-\frac{1}{\underline{9}}\right]
\end{aligned}
$$

The two underlined numbers have to be the same to maintain Lorentz covariance. Next we study how HV corrects the problem.

The triangle diagrams in the light cone gauge is linearly divergent, but the same procedure in sec.2 applies

$$
\begin{aligned}
\Gamma\left(g^{i}, g^{j}, g^{k} ; g\right) & =-\left[\frac{2}{2-D} \frac{-2(12+5 D)}{3 D}+\frac{14}{3}\right]\left\{\delta^{i k} \frac{1}{p_{2}^{+}} K_{21}^{j}+\delta^{j k} \frac{1}{p_{1}^{+}} K_{21}^{i}+\delta^{i j} \frac{1}{p_{3}^{+}} K_{21}^{k}\right\}+\text { Art. Div. } \\
\Gamma\left(g^{i}, g^{j}, g^{k} ; q\right) & =-\left[\frac{2}{2-D} \frac{2(2+3 D)}{3 D}-\frac{10}{3}\right]\left\{\delta^{i k} \frac{1}{p_{2}^{+}} K_{21}^{j}+\delta^{j k} \frac{1}{p_{1}^{+}} K_{21}^{i}+\delta^{i j} \frac{1}{p_{3}^{+}} K_{21}^{k}\right\} \\
\Gamma\left(g^{i}, g^{j}, g^{k} ; s\right) & =-\left[\frac{2}{2-D} \frac{2}{3 D}+\frac{1}{3}\right]\left\{\delta^{i k} \frac{1}{p_{2}^{+}} K_{21}^{j}+\delta^{j k} \frac{1}{p_{1}^{+}} K_{21}^{i}+\delta^{i j} \frac{1}{p_{3}^{+}} K_{21}^{k}\right\}
\end{aligned}
$$

The first term in each bracket is what we get by honestly computing in $D$ dimension, the second terms is due to some peculiarities in using the $\delta$-regulator to evaluate a linearly divergent diagram. An interested 
reader may refer to [19] (Eq.108 in particular) for details. We also give

$$
\begin{aligned}
& \Gamma\left(g^{i}, g^{j},+; s\right)=-\left(p_{1}^{+}-p_{2}^{+}\right) \delta^{i j} \frac{2}{2-D}\left[\frac{D}{12}-\frac{1}{3}\right] \\
& \Gamma\left(g^{i}, g^{j},+; q\right)=-\left(p_{1}^{+}-p_{2}^{+}\right) \delta^{i j} \frac{2}{2-D}\left[-\frac{D}{3}+\frac{4}{3}\right]
\end{aligned}
$$

$\Gamma\left(g^{i}, g^{j}, g^{k} ; g\right)$ and $\Gamma\left(s, s, g^{k}\right)$ contain gauge artificial divergences, which must be cancelled when the self-mass diagrams on the external legs are included.

After extracting the finite parts from above, and combining them with Eq.15, both the $-5 / 18$ and $-1 / 9$ become $-4 / 9$, restoring gauge covariance.

The fermion vertex correction is more complicated. It can be separated into one part proportional to $\gamma^{\mu}$, which is used to compute $Z_{1}$, and another part of the form $\gamma^{i} \gamma^{+} \not p$ that vanishes on-shell

$$
\begin{aligned}
V\left(q, \bar{q}, g^{i}\right)= & -\left(\frac{2}{2-D} \frac{(D+2)(D-8)}{4 D}+\frac{3}{2}\right)\left(\gamma^{+} \frac{q^{i}}{q^{+}}-\gamma^{i}\right) \\
& -\left(\frac{2}{2-D} \frac{D^{2}+2 D-16}{8 D}+\frac{3}{4}\right)\left(\gamma^{i} \gamma^{+} \frac{\not p_{1}}{p_{1}^{+}}-\frac{\not p_{2}}{p_{2}^{+}} \gamma^{i} \gamma^{+}\right)+\text {Art. Div. }
\end{aligned}
$$

The first term is the tree vertex $\left(\epsilon_{i}\right.$ dotted into $\left.\gamma^{\mu}\right)$, so we will take its coefficient to be the correction to $1 / Z_{1}$, without a need to interpret the gamma matrices in non-integer dimension.

\subsection{Assembling}

In $[11,12]$, the helicity conserving gluon amplitudes are computed with the $\delta$-regulator. We found that the results have a lot of mismatches such as a lonesome four-point vertex without the corresponding exchange diagrams to complete to a tree. In [13], when the external states are no longer restricted to be gluons, the mismatches become more colorful, sometimes an entire s or t-channel diagram is hanging. When there are only four particles involved in a (helicity conserving) scattering, the tree amplitude is the only quantity that satisfies the helicity requirement and is Lorentz invariant. These can be deduced either through applying supersymmetry operator to the S-matrix [18] or by brute force exhausting all spinor expressions one can write down.

We next illustrate schematically how HV scheme corrects the mismatch in gluon scattering. The results from $[11,12]$ stand thus (omitting $i g^{4} N_{c} /\left(8 \pi^{2}\right) \operatorname{Tr}\left[t^{a} t^{b} t^{c} t^{d}\right]$ )

$$
\begin{aligned}
A(g-, g-, g+, g+)_{\text {amputate }}= & \frac{-2 K_{12}^{\wedge} p_{3}^{+} p_{4}^{+}}{K_{43}^{\wedge} K_{32}^{\wedge} K_{21}^{\wedge} K_{14}^{\wedge} p_{1}^{+} p_{2}^{+}}\left[-\left(\log ^{2} \frac{s}{t}+\pi^{2}\right)-\frac{11}{3} \log \delta e^{\gamma} t+\frac{73}{9}+\mathrm{IR}\right] \\
& -\frac{1}{3}\left(-2 \frac{p_{1}^{+} p_{3}^{+}+p_{2}^{+} p_{4}^{+}}{\left(p_{1}^{+}+p_{4}^{+}\right)\left(p_{2}^{+}+p_{3}^{+}\right)}\right)+\frac{2}{3}
\end{aligned}
$$

The first line is proportional to a tree while the second line is a multiple of four point vertex and a pure number.

As stated in the earlier sections, we should compute all the superficially divergent parts in this amplitude in $2+\mathrm{D}$ dimension, and keep the $1 / \epsilon$ poles to the very end

$$
\text { Superficially divergent }=-\frac{11}{3}\left[1 / \epsilon+\log \mu^{2}\right] \operatorname{Tree}(2+D)+\text { finite }
$$

Now we identify the first term as $11 / 3 \log \delta$. Tree(4). Those parts that remain finite as $\epsilon \rightarrow 0$ should give $A_{H V}-A_{\delta}$, and we can evaluate them by taking the dimension back to 4 and go to a helicity basis

$$
A_{H V}-A_{\delta}=\frac{2}{3} \frac{p_{1}^{+} p_{3}^{+}+p_{2}^{+} p_{4}^{+}}{\left(p_{1}^{+}+p_{4}^{+}\right)^{2}}-\frac{2}{3} \frac{-2 K_{12}^{\wedge} p_{3}^{+} p_{4}^{+}}{K_{43}^{\wedge} K_{32}^{\wedge} K_{21}^{\wedge} K_{14}^{\wedge} p_{1}^{+} p_{2}^{+}}-\frac{2}{3}
$$

This is to be added to the $\delta$-regulator result, we see that the hanging four point vertex and the pure number are cancelled. 


\subsection{Dimensional Reduction in the Light Cone}

With the dimensional reduction scheme, all the tensor algebra or gamma matrix algebra are performed in $4 \mathrm{D}$, while only the first $4-2 \epsilon$ components of all the momenta are non-zero. To obtain the DR results, we can either insert $(2 \pi)^{2 \epsilon} \delta^{2 \epsilon}(q)$ into the momentum integral or build up from the HV results by including a gluon whose polarity is in between $D$ and 2 . Its polarization vector is

$$
\epsilon_{i}^{\mu}=\left(0, \ldots, \delta_{i}^{\mu}, \ldots, 0\right) ; i \in[D, 2]
$$

actually this gluon behaves in most aspects like an adjoint scalar (it was called $\epsilon$-scalar in [16]). For example, the four gluon box with DR scheme up to terms that vanish as $D \rightarrow 2$ is

$$
B\left(g^{i}, g^{j}, g^{k}, g^{l} ; g\right)=\frac{2}{2-D}\left\{\left[\delta^{i l} \delta^{j k}+\delta^{i j} \delta^{k l}\right] \frac{1}{6}+\delta^{i k} \delta^{j l} \frac{10-3 D}{6}+\text { Art. Div. }\right\}
$$

This result can be obtained by combining $B\left(g^{i}, g^{j}, g^{k}, g^{l} ; g\right)$ and $B\left(g^{i}, g^{j}, g^{k}, g^{l} ; s\right)(2-D)$ from Eq.10. $B\left(g^{i}, g^{j}, g^{k}, g^{l} ; q\right)$ and $B\left(g^{i}, g^{j}, g^{k}, g^{l} ; s\right)$ remains the same in DR. Here we have assumed that the external gluon polarization is in $4-2 \epsilon$ dimension, for a general external polarization, the results are given in the appendix. In fact, the calculations there are done with the tensor algebra or gamma matrix algebra in $D$ dimension and the momentum integral done in $D_{1}$ dimension. Then taking $D=2, D_{1}=2-2 \epsilon$ corresponds to DR and taking $D=D_{1}=2-2 \epsilon$ corresponds to HV.

\section{List of Amplitudes}

The scalars and fermions in the following amplitudes are regrettably given only in the adjoint representation. It turned out not so trivial to go to a general representation. For example, for the amplitude of quark pair production $A\left(g^{a}, g^{b}, q^{i}, \bar{q}_{j}\right)$, the procedure of colour stripping gives the following structure $\left(t^{a} t^{b}\right)_{j}^{i},\left(t^{b} t^{a}\right)_{j}^{i}$ and $\operatorname{Tr}\left(t^{a} t^{b}\right) \delta_{j}^{i}$. So it is necessary to include the diagrams where the two gluons legs are crossed, and thereby lose planarity. Looking back to our calculation done in $[8,11-13]$, the detailed cancellation of the gauge artificial divergence depended rather delicately on having a planar representation of the Feynman diagrams. It is only after the cancellation of the artificial divergence do we find the amplitude together with its infrared terms symmetric under crossing.

The one loop amplitudes are all proportional to the tree level

$$
A_{1}=\frac{-g^{2} N_{c}}{16 \pi^{2}} A_{0} \times F
$$

and in each expression of $F$ in the list, the upper entry in the array applies to HV and the lower to DR scheme.

$$
\begin{aligned}
F(g-, g-, g+, g+)= & {\left[\begin{array}{c}
67 / 9 \\
64 / 9
\end{array}\right]-\frac{4}{9} N_{s}-\frac{20}{9} N_{f} } \\
& {\left[-\frac{11}{3}+\frac{1}{6} N_{s}+\frac{4}{3} N_{f}\right]\left[-\frac{1}{\epsilon}+\log \frac{t e^{\gamma}}{\mu^{2}}\right]-\left(\log ^{2} \frac{s}{t}+\pi^{2}\right) } \\
A(g-, g-, g+, g+)_{0}= & -2 i g^{2} \operatorname{Tr}\left(t^{a} t^{b} t^{c} t^{d}\right) \frac{-2 K_{12}^{\wedge 4} p_{3}^{+} p_{4}^{+}}{K_{43}^{\wedge} K_{32}^{\wedge} K_{21}^{\wedge} K_{14}^{\wedge} p_{1}^{+} p_{2}^{+}} \rightarrow-2 i g^{2} \operatorname{Tr}\left(t^{a} t^{b} t^{c} t^{d}\right) \frac{-2\langle 1 \mid 2\rangle}{\langle 1 \mid 2\rangle\langle 2 \mid 3\rangle\langle 3 \mid 4\rangle\langle 4 \mid 1\rangle} \\
F(g-, g+, g-, g+)= & {\left[\begin{array}{l}
67 / 9 \\
64 / 9
\end{array}\right]-\frac{4}{9} N_{s}-\frac{20}{9} N_{f}+\frac{s t\left(2+N_{s}-4 N_{f}\right)}{2(s+t)^{2}} } \\
& +\left[-\frac{11}{3}+\frac{1}{6} N_{s}+\frac{4}{3} N_{f}\right]\left[-\frac{1}{\epsilon}+\log \frac{s e^{\gamma}}{\mu^{2}}\right]+\frac{s}{6(s+t)^{3}} \log \frac{s}{t} \times \\
& {\left[\left(28 t^{2}+38 s t+22 s^{2}\right)+N_{s}\left(2 t^{2}-5 s t-s^{2}\right)-N_{f}\left(8 s^{2}+4 s t+20 t^{2}\right)\right] }
\end{aligned}
$$




$$
\begin{gathered}
-\frac{1}{2(t+s)^{4}}\left[2\left(s^{2}+s t+t^{2}\right)^{2}+2 N_{f}\left(s t^{3}+s^{3} t\right)+N_{s} s^{2} t^{2}\right]\left(\log ^{2} \frac{s}{t}+\pi^{2}\right) \\
A(g-, g+, g-, g+)_{0}=-2 i g^{2} \operatorname{Tr}\left(t^{a} t^{b} t^{c} t^{d}\right) \frac{-2 K_{13}^{\wedge} p_{2}^{+} p_{4}^{+}}{K_{43}^{\wedge} K_{32}^{\wedge} K_{21}^{\wedge} K_{14}^{\wedge} p_{1}^{+} p_{3}^{+}} \rightarrow-2 i g^{2} \operatorname{Tr}\left(t^{a} t^{b} t^{c} t^{d}\right) \frac{-2\langle 1 \mid 3\rangle^{4}}{\langle 1 \mid 2\rangle\langle 2 \mid 3\rangle\langle 3 \mid 4\rangle\langle 4 \mid 1\rangle}
\end{gathered}
$$

$$
\begin{aligned}
& F(s, s, g-, g+)=\left[\begin{array}{l}
8 \\
8
\end{array}\right]-4\left[-\frac{1}{\epsilon}+\log \frac{s e^{\gamma}}{\mu^{2}}\right]+\frac{2 s}{s+t} \log \frac{s}{t}-\frac{s^{2}+s t+t^{2}}{(s+t)^{2}}\left(\log ^{2} \frac{s}{t}+\pi^{2}\right) \\
& A(s, s, g-, g+)_{0}=-2 i g^{2} \operatorname{Tr}\left(t^{a} t^{b} t^{c} t^{d}\right) \frac{-8 K_{13}^{\wedge 2} K_{14}^{\vee 2}}{s t p_{3}^{+} p_{4}^{+} p_{1}^{+2}} \rightarrow-2 i g^{2} \operatorname{Tr}\left(t^{a} t^{b} t^{c} t^{d}\right) \frac{-2\langle 3 \mid 1\rangle^{2}[1 \mid 4]^{2}}{s t} \\
& F(g-, s, g+, s)=\left[\begin{array}{l}
8 \\
8
\end{array}\right]-4\left[-\frac{1}{\epsilon}+\log \frac{s e^{\gamma}}{\mu^{2}}\right]+2 \log \frac{s}{t}-\left(\log ^{2} \frac{s}{t}+\pi^{2}\right) \\
& A(g-, s, g+, s)_{0}=-2 i g^{2} \operatorname{Tr}\left(t^{a} t^{b} t^{c} t^{d}\right) \frac{-8 K_{21}^{\wedge 2} K_{32}^{\vee 2}}{s t p_{1}^{+} p_{3}^{+} p_{2}^{+2}} \rightarrow-2 i g^{2} \operatorname{Tr}\left(t^{a} t^{b} t^{c} t^{d}\right) \frac{-2\langle 1 \mid 2\rangle^{2}[3 \mid 2]^{2}}{s t} \\
& F(s, s, q-, \bar{q}-)=\left[\begin{array}{c}
68 / 9 \\
62 / 9
\end{array}\right]+\frac{4}{9} N_{s}+\frac{20}{9} N_{f} \\
& -\left[\frac{10}{3}+\frac{1}{6} N_{s}+\frac{4}{3} N_{f}\right]\left[-\frac{1}{\epsilon}+\log \frac{s e^{\gamma}}{\mu^{2}}\right]-\frac{2 t+s}{2(s+t)}\left(\log ^{2} \frac{s}{t}+\pi^{2}\right) \\
& A(s, s, q-, \bar{q}-)_{0}=-2 i g^{2} \operatorname{Tr}\left(t^{a} t^{b} t^{c} t^{d}\right) \frac{4 K_{13}^{\wedge} K_{14}^{\vee}}{s p_{1}^{+} p_{3}^{+}} \rightarrow-2 i g^{2} \operatorname{Tr}\left(t^{a} t^{b} t^{c} t^{d}\right) \frac{2\langle 3 \mid 1\rangle[1 \mid 4]}{s} \\
& A\left(s_{\alpha}, s_{\beta}, s_{\beta}, s_{\alpha}\right)_{1}=\frac{i g^{4} N_{c}}{8 \pi^{2}} \operatorname{Tr}\left(t^{a} t^{b} t^{c} t^{d}\right)\left[\frac{1}{9}\left[\begin{array}{c}
95+154 s / t \\
107+160 s / t
\end{array}\right]\right. \\
& +\left(\frac{2 s}{t}+1\right)\left[\frac{4}{9} N_{s}+\frac{20}{9} N_{f}-\left(\frac{1}{6} N_{s}+\frac{4}{3} N_{f}\right)\left[-\frac{1}{\epsilon}+\log \frac{t e^{\gamma}}{\mu^{2}}\right]\right] \\
& \left.-\frac{2(13 s+8 t)}{3 t}\left[-\frac{1}{\epsilon}+\log \frac{t e^{\gamma}}{\mu^{2}}\right]-\frac{1}{2}\left[-\frac{1}{\epsilon}+\log \frac{s e^{\gamma}}{\mu^{2}}\right]-\frac{2 s}{t}\left(\log ^{2} \frac{s}{t}+\pi^{2}\right)\right] \\
& A\left(s_{\alpha}, s_{\beta}, s_{\beta}, s_{\alpha}\right)_{0}=-2 i g^{2} \operatorname{Tr}\left(t^{a} t^{b} t^{c} t^{d}\right) \frac{2 s+t}{t} ;(\alpha \neq \beta) \\
& F(g-, g+, q-, \bar{q}-)=\left[\begin{array}{l}
7 \\
6
\end{array}\right]-3\left[-\frac{1}{\epsilon}+\log \frac{s e^{\gamma}}{\mu^{2}}\right]+\frac{3 s}{s+t} \log \frac{s}{t}-\frac{2 s^{2}+s t+2 t^{2}}{2(s+t)^{2}}\left(\log ^{2} \frac{s}{t}+\pi^{2}\right) \\
& A(g-, g+, q-, \bar{q}-)_{0}=-2 i g^{2} \operatorname{Tr}\left(t^{a} t^{b} t^{c} t^{d}\right) \frac{-8 K_{13}^{\wedge 2} K_{32}^{\vee} K_{24}^{\vee}}{s t p_{1}^{+} p_{2}^{+} p_{3}^{+2}} \rightarrow-2 i g^{2} \operatorname{Tr}\left(t^{a} t^{b} t^{c} t^{d}\right) \frac{-2\langle 3 \mid 1\rangle^{2}[3 \mid 2][2 \mid 4]}{s t} \\
& F(g-, g+, q+, \bar{q}+)=\left[\begin{array}{l}
7 \\
6
\end{array}\right]-3\left[-\frac{1}{\epsilon}+\log \frac{s e^{\gamma}}{\mu^{2}}\right]-\left(\log ^{2} \frac{s}{t}+\pi^{2}\right) \\
& A(g-, g+, q+, \bar{q}+)_{0}=-2 i g^{2} \operatorname{Tr}\left(t^{a} t^{b} t^{c} t^{d}\right) \frac{8 K_{14}^{\wedge} K_{13}^{\wedge} K_{32}^{\vee 2}}{s t p_{1}^{+} p_{2}^{+} p_{3}^{+2}} \rightarrow-2 i g^{2} \operatorname{Tr}\left(t^{a} t^{b} t^{c} t^{d}\right) \frac{2\langle 4 \mid 1\rangle\langle 3 \mid 1\rangle[3 \mid 2]^{2}}{s t} \\
& A(g-, q-, g+, \bar{q}-)=\left[\begin{array}{l}
7 \\
6
\end{array}\right]-3\left[-\frac{1}{\epsilon}+\log \frac{t e^{\gamma}}{\mu^{2}}\right]-\left(\log ^{2} \frac{s}{t}+\pi^{2}\right) \\
& A(g-, q-, g+, \bar{q}-)_{0}=-2 i g^{2} \operatorname{Tr}\left(t^{a} t^{b} t^{c} t^{d}\right) \frac{-8 K_{21}^{\wedge 2} K_{43}^{\vee} K_{32}^{\vee}}{s t p_{1}^{+} p_{3}^{+} p_{2}^{+2}} \rightarrow-2 i g^{2} \operatorname{Tr}\left(t^{a} t^{b} t^{c} t^{d}\right) \frac{-2\langle 1 \mid 2\rangle^{2}[4 \mid 3][3 \mid 2]}{s t}
\end{aligned}
$$




$$
\begin{aligned}
F\left(q_{\alpha}-, q_{\beta}-, \bar{q}_{\beta}-, \bar{q}_{\alpha}-\right)= & {\left[\begin{array}{c}
59 / 9 \\
44 / 9
\end{array}\right]+\frac{4}{9} N_{s}+\frac{20}{9} N_{f}-\left[\frac{7}{3}+\frac{1}{6} N_{s}+\frac{4}{3} N_{f}\right]\left[-\frac{1}{\epsilon}+\log \frac{t e^{\gamma}}{\mu^{2}}\right] } \\
& -\left(\log ^{2} \frac{s}{t}+\pi^{2}\right) \\
A\left(q_{\alpha}-, q_{\beta}-, \bar{q}_{\beta}-, \bar{q}_{\alpha}-\right)_{0}= & -2 i g^{2} \operatorname{Tr}\left(t^{a} t^{b} t^{c} t^{d}\right) \frac{-4 K_{21}^{\wedge} K_{43}^{\vee}}{t p_{1}^{+} p_{2}^{+}} \rightarrow-2 i g^{2} \operatorname{Tr}\left(t^{a} t^{b} t^{c} t^{d}\right) \frac{-2\langle 1 \mid 2\rangle[4 \mid 3]}{t} ;(\alpha \neq \beta) \\
F\left(q_{\alpha}-, \bar{q}_{\beta}-, q_{\beta}-, \bar{q}_{\alpha}-\right)= & {\left[\begin{array}{c}
59 / 9 \\
44 / 9
\end{array}\right]+\frac{4}{9} N_{s}+\frac{20}{9} N_{f}-\left[\frac{7}{3}+\frac{1}{6} N_{s}+\frac{4}{3} N_{f}\right]\left[-\frac{1}{\epsilon}+\log \frac{t e^{\gamma}}{\mu^{2}}\right] } \\
& -\frac{t}{s+t} \log \frac{s}{t}-\frac{2 s^{2}+s t+2 t^{2}}{2(s+t)^{2}}\left(\log ^{2} \frac{s}{t}+\pi^{2}\right) \\
A\left(q_{\alpha}-, \bar{q}_{\beta}-, q_{\beta}-, \bar{q}_{\alpha}-\right)_{0}= & -2 i g^{2} \operatorname{Tr}\left(t^{a} t^{b} t^{c} t^{d}\right) \frac{4 K_{13}^{\wedge} K_{24}^{\vee}}{t p_{1}^{+} p_{3}^{+}} \rightarrow-2 i g^{2} \operatorname{Tr}\left(t^{a} t^{b} t^{c} t^{d}\right) \frac{2\langle 3 \mid 1\rangle[2 \mid 4]}{t} ;(\alpha \neq \beta) \\
F\left(q_{\alpha}-, \bar{q}_{\alpha}-, q_{\beta}-, \bar{q}_{\beta}-\right)= & {\left[\begin{array}{c}
59 / 9 \\
44 / 9
\end{array}\right]+\frac{4}{9} N_{s}+\frac{20}{9} N_{f}-\left[\frac{7}{3}+\frac{1}{6} N_{s}+\frac{4}{3} N_{f}\right]\left[-\frac{1}{\epsilon}+\log \frac{s e^{\gamma}}{\mu^{2}}\right] } \\
& +\frac{s}{s+t} \log \frac{s}{t}-\frac{2 s^{2}+s t+2 t^{2}}{2(s+t)^{2}}\left(\log ^{2} \frac{s}{t}+\pi^{2}\right) \\
A\left(q_{\alpha}-, \bar{q}_{\alpha}-, q_{\beta}-, \bar{q}_{\beta}-\right)_{0}= & -2 i g^{2} \operatorname{Tr}\left(t^{a} t^{b} t^{c} t^{d}\right) \frac{4 K_{13}^{\wedge} K_{24}^{\vee}}{s p_{1}^{+} p_{3}^{+}} \rightarrow-2 i g^{2} \operatorname{Tr}\left(t^{a} t^{b} t^{c} t^{d}\right) \frac{2\langle 3 \mid 1\rangle[2 \mid 4]}{s} ;(\alpha \neq \beta) \quad(27)
\end{aligned}
$$

In giving the tree amplitude, we have converted the $K_{i j}$ into the normalized spinors to conform to the fashion. The reader may find that in an amplitude involving fermions the factors of $p^{+}$is not what is needed to convert the $K_{i j}$ into the spinor products. The reason is merely for the sake of automated calculation. The correct external line factor is $\sqrt{\sqrt{2} p_{i}^{+}}$for every fermion line, but in order to avoid the phase ambiguity, we only associate $\sqrt{2} p_{i}^{+}$to an outgoing fermi line.

\section{$4 \quad \mathrm{~N}=1$ Supersymmetry}

One can go to $N=1$ gauge multiplet by taking $N_{s}=0$ and $N_{f}=1 / 2$. The amputated amplitudes with DR are given by

$$
\begin{aligned}
& A(-,-,+,+)=\text { tree }_{1}\left[6-3\left[-\frac{1}{\epsilon}+\log \frac{t e^{\gamma}}{\mu^{2}}\right]-\left(\log ^{2} \frac{s}{t}+\pi^{2}\right)\right] \\
& A(-,+,-,+)=\text { tree }_{2}\left[6-3\left[-\frac{1}{\epsilon}+\log \frac{s e^{\gamma}}{\mu^{2}}\right]+\frac{3 s}{(s+t)} \log \frac{s}{t}-\frac{\left(2 s^{2}+s t+2 t^{2}\right)}{2(t+s)^{2}}\left(\log ^{2} \frac{s}{t}+\pi^{2}\right)\right]
\end{aligned}
$$

From Eq.52, the self-mass is already supersymmetric, we need only check the supersymmetry relations among the tree amplitudes here. The covariant treatment can be found in [20]. Here we stick to the light cone and write down the trees with the light cone superfields. Indeed, the two amplitudes above correspond to $A(\phi, \phi, \bar{\phi}, \bar{\phi})$ and $A(\phi, \bar{\phi}, \phi, \bar{\phi})$ respectively. Referring to the appendix, the four point vertex can be absorbed into the exchange diagrams, so only three graphs contribute to the first amplitude (a factor of $-2 g^{2} \operatorname{Tr}\left[t^{a} t^{b} t^{c} t^{d}\right]$ is understood to multiply the following)

Fig.1 gives

$$
\begin{aligned}
A(\phi, \phi, \bar{\phi}, \bar{\phi}) & =i \int d \theta\left\{\left(D \bar{\phi}_{4}\right) \phi_{1} D\left(\bar{\phi}_{3} D \phi_{2}\right)\langle 1 \mid 4\rangle[4 \mid 3] \frac{p_{4}^{+}}{t\left(p_{1}+p_{4}\right)^{+}}\right. \\
& \left.+\quad\left(D \bar{\phi}_{3}\right) \phi_{2} D\left(\bar{\phi}_{4} D \phi_{1}\right)\langle 2 \mid 3\rangle[3 \mid 4] \frac{p_{3}^{+}}{t\left(p_{2}+p_{3}\right)^{+}}-D\left(\bar{\phi}_{4} \bar{\phi}_{3}\right) \phi_{1} \phi_{2}\langle 1 \mid 2\rangle[3 \mid 4] \frac{2\left(p_{1}^{+}+p_{2}^{+}\right)}{s}\right\}
\end{aligned}
$$




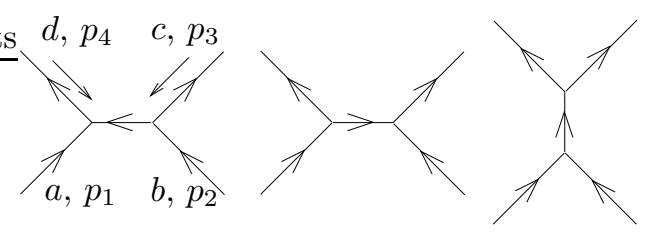

Figure 1: The three Feynman diagrams that contribute to $A(\phi, \phi, \bar{\phi}, \bar{\phi})$, incoming (outgoing) arrow corresponds to $\phi(\bar{\phi})$

where $\phi_{i}$ means $\phi\left(p_{i} ; \theta\right)$ and the spinors are unnormalized spinors. We can further simplify the above to get

$$
i D\left\{D\left(\bar{\phi}_{4} \bar{\phi}_{3}\right) \phi_{1} \phi_{2} \frac{\langle 12\rangle[34]}{s t\left(p_{1}+p_{2}\right)^{+}}\left[-4 p_{1}^{+} p_{2}^{+} p_{3}^{+} p_{4}^{+}\langle 12\rangle[34]-2 s p_{1}^{+} p_{3}^{+}\right]-\left(D \bar{\phi}_{3}\right) \phi_{2} D\left(\bar{\phi}_{4} D \phi_{1}\right) \frac{\langle 12\rangle[34]}{t}\right\} \mid
$$

By inserting the above in between definite incoming and outgoing asymptotic states, one can check that it does give the correct component amplitudes. In fact the verification is trivial for the case of four gluon and four gluino scattering. The verification of two gluon and two gluino amplitude is a bit involved. As most readers are not familiar with manipulating light cone spinors, we shall demonstrate it as an example. If we aim for $A(q-, g-, g+, \bar{q}-)$, then we arrange all the $D$ 's to act on $\phi_{1}$ and $\bar{\phi}_{4}$

$$
\begin{aligned}
A(q-, g-, g+, \bar{q}-) & =\left\{-\left(D \bar{\phi}_{4}\right) \bar{\phi}_{3}\left(D \phi_{1}\right) \phi_{2} \frac{i\langle 12\rangle[34]}{s t\left(p_{1}+p_{2}\right)^{+}}\left[-4 p_{1}^{+} p_{2}^{+} p_{3}^{+} p_{4}^{+}\langle 12\rangle[34]-2 s p_{1}^{+} p_{3}^{+}\right]\right. \\
& \left.-\left(2 p_{3}^{+} \bar{\phi}_{3}\right) \phi_{2}\left(D \bar{\phi}_{4}\right)\left(D \phi_{1}\right) \frac{i\langle 12\rangle[34]}{t}\right\} \mid \\
& =-\sqrt{2} \bar{\psi}_{4} A_{3}^{\wedge} \psi_{1} A_{2}^{\vee}\left\{-\frac{i\langle 12\rangle^{2}[34]^{2}}{s t\left(p_{1}+p_{2}\right)^{+}} 4 p_{1}^{+} p_{2}^{+} p_{3}^{+} p_{4}^{+}+2 p_{2}^{+} p_{3}^{+} \frac{i\langle 12\rangle[34]}{t\left(p_{1}+p_{2}\right)^{+}}\right\}
\end{aligned}
$$

Use the identity

$$
p_{1}^{+} p_{2}^{+} p_{4}^{+}\langle 2 \mid 1\rangle=\left(p_{1}+p_{2}\right)^{+} p_{2}^{+} p_{4}^{+}\langle 2 \mid 4\rangle-p_{2}^{+} p_{3}^{+} p_{4}^{+}\langle 4 \mid 3\rangle
$$

to swap one of the $\langle 12\rangle$ in the first term of Eq.30

$$
\begin{aligned}
\frac{i\langle 12\rangle^{2}[34]^{2}}{s t\left(p_{1}+p_{2}\right)^{+}} 4 p_{1}^{+} p_{2}^{+} p_{3}^{+} p_{4}^{+} & =\frac{i\langle 12\rangle[34]^{2}}{s t\left(p_{1}+p_{2}\right)^{+}} 4 p_{3}^{+}\left[-\left(p_{1}+p_{2}\right)^{+} p_{2}^{+} p_{4}^{+}\langle 2 \mid 4\rangle+p_{2}^{+} p_{3}^{+} p_{4}^{+}\langle 4 \mid 3\rangle\right] \\
& =\frac{-4 i\langle 12\rangle\langle 24\rangle[34]^{2}}{s t} p_{3}^{+} p_{2}^{+} p_{4}^{+}+\frac{2 i\langle 12\rangle[34]}{s t\left(p_{1}+p_{2}\right)^{+}} p_{3}^{+} p_{2}^{+}
\end{aligned}
$$

we arrive at

$$
A(q-, g-, g+, \bar{q}-)=-\sqrt{2} \bar{\psi}_{4} A_{3}^{\wedge} \psi_{1} A_{2}^{\vee} \frac{4 i\langle 12\rangle\langle 24\rangle[34]^{2} p_{3}^{+} p_{2}^{+} p_{4}^{+}}{s t}
$$

By inserting the above into incoming and outgoing states, we obtain an extra factor of $\sqrt{2 p_{1}^{+} p_{4}^{+}}$from the free field expansion of $\psi$. So

$$
A(q-, g-, g+, \bar{q}-)=-\frac{8 i\langle 12\rangle\langle 24\rangle[34]^{2} p_{3}^{+} p_{2}^{+} p_{4}^{+} \sqrt{p_{1}^{+} p_{4}^{+}}}{s t} \rightarrow-\frac{2 i\langle 12\rangle\langle 24\rangle[34]^{2}}{s t}
$$

The spinors in the last expression are properly normalized.

Similarly, the amplitude $A(\phi, \bar{\phi}, \phi, \bar{\phi})$ can be written

$$
\begin{aligned}
A(\phi, \bar{\phi}, \phi, \bar{\phi}) & =i D\left\{\left(-\left(D \bar{\phi}_{2}\right) \phi_{1} D\left(\bar{\phi}_{4} D \phi_{3}\right) \frac{1}{s}+\left(D \bar{\phi}_{2}\right) \phi_{3} D\left(\bar{\phi}_{4} D \phi_{1}\right) \frac{1}{t}\right)\langle 1 \mid 3\rangle[2 \mid 4]\right. \\
& \left.-D\left(\bar{\phi}_{2} \bar{\phi}_{4}\right) \phi_{1} \phi_{3} \frac{\langle 1 \mid 3\rangle[2 \mid 4]}{s t\left(p_{1}+p_{3}\right)^{+}}\left(4 p_{1}^{+} p_{2}^{+} p_{3}^{+} p_{4}^{+}\langle 1 \mid 3\rangle[2 \mid 4]-2 s p_{1}^{+} p_{2}^{+}+2 t p_{2}^{+} p_{3}^{+}\right)\right\} \mid
\end{aligned}
$$




\section{$5 \quad$ Infrared Terms}

So far we have been silent about all the infrared terms and external self-mass diagrams. The rigorous treatment of the infrared issue can be found in [11]. Here we merely try to establish the link between the infrared terms regulated with cut off in $q^{+}$and the ones regulated by an almighty $\epsilon$ in the literature.

One can always factor a scattering amplitude into the following form $[22,23]$

$$
A_{n}=J\left(\frac{Q^{2}}{\mu^{2}}, \alpha(\mu)\right) \times S\left(p_{i}, \frac{Q^{2}}{\mu^{2}}, \alpha(\mu)\right) \times h_{n}\left(p_{i}, \frac{Q^{2}}{\mu^{2}}, \alpha(\mu)\right)
$$

where $J$ is the 'jet' function and $S$ is the 'soft' part and $h_{n}$ is the hard remainder, which is finite after renormalization is performed. In a dimensional regulation scheme, the jet function will contain a double pole in $\epsilon$, caused by the overlap of collinear divergence and infrared divergence. In the light cone gauge, it is given by the external self-mass terms. In fact, the collinear divergence shows up as $\log p^{2}$ in Eq.52, and the infrared divergence shows up as the divergent integral in $q^{+} \cdot \log p^{2}$ can be replaced with $\log \left(\Delta^{2}\right)(\Delta$ is the jet resolution) when the collinear emissions are included.

The rest of the infrared terms are interpreted as the soft part $S$ above. They can be cast into the following form

$$
\begin{aligned}
\text { IR term } & =-\frac{i g^{2} N_{c}}{16 \pi^{2}} A_{0} \sum_{i=1 \ldots 4} I\left(p_{i}, p_{i+1}\right) \\
I\left(p_{i}, p_{i+1}\right) & =\int_{0}^{\left|p_{i}^{+}\right|} \frac{d q^{+}}{q^{+}}\left[-\frac{1}{\epsilon}+\log \frac{q^{+2}\left|\left(p_{i}+p_{i+1}\right)^{2}\right| e^{\gamma}}{\mu^{2}\left|p_{i}^{+} p_{i+1}^{+}\right|}\right]+\int_{0}^{\left|p_{i+1}^{+}\right|} \frac{d q^{+}}{q^{+}}\left[-\frac{1}{\epsilon}+\log \frac{q^{+2}\left|\left(p_{i}+p_{i+1}\right)^{2}\right| e^{\gamma}}{\mu^{2}\left|p_{i}^{+} p_{i+1}^{+}\right|}\right] \\
& +\int_{-p_{i}^{+}}^{p_{i+1}^{+}} \frac{d q^{+}}{q^{+}} \log \left|\frac{\left(p_{i+1}^{+}-q^{+}\right) p_{i}^{+}}{\left(q^{+}+p_{i}^{+}\right) p_{i+1}^{+}}\right|
\end{aligned}
$$

First of all, the appearance of $\epsilon$ in Eq.34 is temporary, our IR singularity is not regulated by $\epsilon$. The form of the infrared terms are slightly asymmetric: the third integral might or might not cross over 0 depending on the sign of the momenta, giving a difference proportional to $\pi^{2}$. We have not computed the amplitudes with quarks in the fundamental representation, but we believe that in general there is going to be one such $I\left(p_{i}, p_{i+1}\right)$ for the region between particle $\mathrm{i}$ and $\mathrm{i}+1$ if it is bounded by a color line. So for the amplitude fig. 2 ,

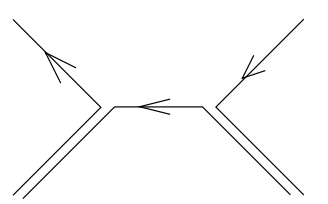

Figure 2: The double line notation for the quark pair production amplitude

there will be $I\left(p_{1}, p_{2}\right), I\left(p_{2}, p_{3}\right)$ and $I\left(p_{4}, p_{1}\right)$ but no $I\left(p_{3}, p_{4}\right)$. The reason is that these IR terms are nothing but Sudakov form factors, which is the square root of the photon to quark anti-quark pair amplitude.

We need to establish the dictionary between the light cone infrared terms and the dimensionally regulated infrared term. This is simply achieved by subtracting the amplitudes given in sec.3 by the corresponding ones obtained with the coventional dimensional regulation. The non-trivial point is that the dictionary thus obtained should be universal. The amplitude for MSYM is the simplest, it is given by

$$
A(g, g, g, g ; \mathrm{MSYM})=\frac{i g^{2} N_{c}}{16 \pi^{2}}\left(\log ^{2} \frac{s}{t}+\pi^{2}\right) A_{\text {tree }}+\text { ext self-mass }+ \text { IR terms }
$$

the same is given in [5] with dimensional reduction

$$
A(g, g, g, g ; \mathrm{MSYM})=-\frac{1}{2} s t I_{4}^{(1)}(s, t) \frac{i g^{2} N_{c}}{8 \pi^{2}} A_{t r e e}
$$




$$
=\frac{i g^{2} N_{c}}{8 \pi^{2}}\left[-\frac{2}{\epsilon^{2}}+\frac{1}{\epsilon} \log \frac{s t}{\mu^{4}}-\log \frac{s}{\mu^{2}} \log \frac{t}{\mu^{2}}+\frac{2 \pi^{2}}{3}\right] A_{\text {tree }}
$$

The difference of the two expressions above gives the correspondence between the infrared terms

$$
\begin{aligned}
\text { Eq.34 }= & -\frac{\pi^{2}}{3}+\frac{4}{\epsilon^{2}}+\frac{2}{\epsilon} \log \frac{\mu^{4}}{s t}+\log ^{2} \frac{\mu^{2}}{s}+\log ^{2} \frac{\mu^{2}}{t} \\
& +\sum_{i} \int_{0}^{p_{i}^{+}} \frac{d q^{+}}{p_{i}^{+}}\left[\frac{-2}{p_{i}^{+}-q^{+}}+\frac{-2}{q^{+}}\right]\left[-\frac{1}{\epsilon}+\log \frac{\left(p_{i}^{+}-q^{+}\right) q^{+} \Delta^{2} e^{\gamma}}{\mu^{2} p_{i}^{+2}}\right]
\end{aligned}
$$

Note we can reshuffle the $1 / \epsilon$ terms from the last term (external self-mass terms) on the rhs to lhs. In fact, they precisely cancel, as promised underneath Eq.34. We can use the dictionary Eq.37 to compare the rest of our results in sec. 3 with those in the literature.

Eq.37 is also interesting in the sense that it can be checked by computing a light-like Wilson loop with cusps, but with the infrared divergence regulated with our scheme.

\section{Concluding Remarks}

To summarize, in this article we focused on obtaining the difference in the amplitudes between HV, DR and the $\delta$-regulator. The computation is done in a cartesian basis, contrary to what is in fashion. The dimensional parameter $\epsilon$ is only used to regulate the UV, while the IR is regulated by cutting off $q^{+}$, as was done in our earlier works. In the context of $N=4 \mathrm{SYM}$, this means the total disappearance of $\epsilon$. In this aspect, our regularization scheme is quite different from the conventional dimensional regularization schemes, put in less accurate words, our calculation is as close to 4D as it can get and yet still preserves gauge symmetry. The supersymmetric identities in $N=1$ SUSY between various amplitudes can be easily checked. The results using HV of course shows SUSY violation.

The IR terms thus obtained is universal as they should be, in fact, we see from Eq.37 that can be interpreted as the Sudakov form factor. As a subject for further investigation, we would like to understand the correspondence Eq.37 better. On one hand our IR terms are well 'localized', that is, involves two neighboring legs at a time, on the other hand, we know in conventional dimensional regulation the external self-mass terms vanish and the IR singularity comes from a single scalar box integral

$$
I_{4}^{(1)}(s, t)=-i e^{\epsilon \gamma} \pi^{-(2-\epsilon)} \int \frac{d^{4-2 \epsilon} q}{q^{2}\left(q-p_{1}\right)^{2}\left(q-p_{1}-p_{2}\right)^{2}\left(q+p_{4}\right)^{2}}
$$

We have not yet studied how to obtain the correspondence Eq.37 from a presumably short calculation. In $[23,24]$, it was shown that this form factor exponentiates, which is a prototype of the BDS iteration relation. With the current data in the light cone at hand, we are far from being able to study the exponentiation of the IR terms. One possible way to proceed is to clarify the correspondence Eq.37 further and convert the IR term from the literature into light cone form. As was mentioned in the introduction, we can shuffle finite terms among the three factors in Eq.33. With our simple minded infrared regulation, the hard remainder $h_{n}$ (listed in sec.3) is fortunately Lorentz covariant. We take it as a good sign that the factorization ordained by our infrared regulation scheme will give a hard remainder that also obeys a nice iteration relation similar to [5]. It is presumably quite hard to push the light cone computation to high loops, no matter how much we advertise it, it is not the best scheme to do calculations with. Alternative routes are yet to be found.

Acknowledgements The author would like to thank prof C.B.Thorn for both initiating the current work and giving a lot of helpful consultations along the way. The work is supported by the grant for research of the Department of Theoretical Physics, Uppsala University.

\section{A Notation and Convention}

Metric in this article is $g^{\mu \nu}=\operatorname{diag}(1,-1,-1,-1)$. 
Polarization vectors are given by

$$
\epsilon_{i}^{\mu}=\left(\frac{1}{\sqrt{2}} \frac{k^{i}}{k^{+}}, \ldots, \delta_{i}^{\mu}, \ldots,-\frac{1}{\sqrt{2}} \frac{k^{i}}{k^{+}}\right)
$$

satisfies

$$
\sum_{i} \epsilon_{i}^{\mu} \epsilon_{i}^{\nu}+g^{\mu+} g^{\nu+} \frac{k^{2}}{k^{+2}}=-g^{\mu \nu}+\frac{g^{\mu+} k^{\nu}}{k^{+}}+\frac{g^{\nu+} k^{\mu}}{k^{+}}
$$

The rhs is the numerator of the gluon propagator, so it can be decomposed into a part that describes the transverse polarizations and a part that describes the 'propagation of the $A^{-}$. The $k^{2}$ factor on the lhs will cancel the propagator, which shows that $A^{-}$is non-dynamical. The $1 / k^{+}$factor is what we call 'gauge artificial divergence' throughout the article. The fate of these divergences in a physical quantity is:

- simple and double poles must all cancel

- double poles coincident with a logarithm must cancel

- simple poles coincident with a logarithm must arrange themselves into the universal IR terms Eq.34

Using the polarization vectors we can pass from the covariant YM lagrangian to the light cone gauge fixed lagrangian. A three point vertex in the light cone can be obtained by dotting these polarization vectors into the covariant vertex functions. The results are simply

$$
V^{i j k}=\delta^{i j}\left[\left(p_{1}-p_{2}\right)^{+} \frac{p_{3}^{k}}{p_{3}^{+}}-\left(p_{1}-p_{2}\right)^{k}\right]+\text { cyclic permutation }
$$

By fixing, say, $i=j=\wedge$ and $k=\vee$, we may reduce the three terms above to only one. This shows the advantage of the helicity basis over the cartesian basis.

Spinors in the light cone are given by dotting a $\not p$ into a reference spinor $\eta$.

$$
\begin{aligned}
& |p\rangle=-\frac{1}{\sqrt{2} p^{+}}\left(p_{\mu} \sigma^{\mu}\right)^{a \dot{a}} \eta_{\dot{a}}=-\frac{1}{\sqrt{2} p^{+}} p_{\mu} \sigma^{\mu}\left[\begin{array}{c}
0 \\
-1
\end{array}\right]=\left[\begin{array}{c}
-\frac{p^{\wedge}}{p^{+}} \\
1
\end{array}\right] ;[p|=| p\rangle^{\dagger} \\
& \mid p]=\frac{1}{\sqrt{2} p^{+}}\left(p_{\mu} \bar{\sigma}^{\mu}\right)_{\dot{a} a} \eta^{a}=\frac{1}{\sqrt{2} p^{+}} p_{\mu} \bar{\sigma}^{\mu}\left[\begin{array}{l}
1 \\
0
\end{array}\right]=\left[\begin{array}{c}
1 \\
\frac{p^{\vee}}{p^{+}}
\end{array}\right] ;\langle p|=| p]^{\dagger}
\end{aligned}
$$

These spinors are not properly normalized unless we further multiply them by $2^{1 / 4} \sqrt{p^{+}}$. The spinors appearing in this article are all unnormalized unless stated otherwise.

The quantity $K_{i, j}$ was also used in our previous calculations. They are directly related to the spinor products and we give them here to facilitate the comparison with our earlier works

$$
\begin{aligned}
& K_{q, p}^{\wedge}=\langle p \mid q\rangle p^{+} q^{+}=\left(q^{+} p^{\wedge}-p^{+} q^{\wedge}\right) \\
& K_{p, q}^{\vee}=[p \mid q] p^{+} q^{+}=\left(p^{+} q^{\vee}-q^{+} p^{\vee}\right)
\end{aligned}
$$

we use the unnormalized spinors or the $K$ 's, to avoid the phase ambiguity in $\sqrt{p^{+}}$. Color Stripping decomposes an amplitude into color subamplitudes

$$
M(1,2 \ldots, n)=\Sigma_{\sigma} \operatorname{Tr}\left[t^{\sigma(1)} t^{\sigma(2)} \ldots t^{\sigma(n)}\right] A(\sigma(1), \sigma(2), \ldots \sigma(n))
$$

where $t^{a}$ is in the fundamental representation normalized so that $\operatorname{Tr} t^{a} t^{b}=1 / 2 \delta^{a b}$, and summation is over all the cyclically inequivalent permutation $\sigma$. Since we have restricted ourselves to the fields in the adjoint representation, this procedure is equivalent to picking out all the planar diagrams with the external legs ordered in the specific order given by $\sigma$.

Lastly, the usual factors associated with dimensional regulation such as $(2 \pi)^{-\epsilon}$ from the momentum integration and $2^{-\epsilon}$ from Tr1 will be left out to avoid cluttering the presentation. These factors, if necessary, can be recovered easily. 


\section{B Light cone superfield}

We follow Mandelstam [21] and use only one real Grassmann coordinate for one supersymmetry. Define the super derivative

$$
D=\frac{\partial}{\partial \theta}+2 i \partial^{+} \theta ; \quad D^{2}=2 i \partial^{+}
$$

The superfields are given by

$$
\phi=A^{\vee}+2^{1 / 4} \theta \psi ; \bar{\phi}=A^{\wedge}+2^{1 / 4} \theta \bar{\psi}
$$

$\bar{\phi}$ is not the conjugate of $\phi$. The $\mathrm{N}=1$ gauge multiplet can be packed nicely by using spinor notations

$$
\begin{aligned}
L & =\left[\bar{\phi}^{a}\left|\left\{i \frac{1}{\sqrt{2}} \delta^{a c} \partial \cdot \bar{\sigma}-i g f^{a b c}\left(\frac{1}{2} \mid \bar{\phi}^{b}\right]\langle\eta|+| \eta]\left\langle\phi^{b}\right|\right)\right\}\left|D \phi^{c}\right\rangle\right. \\
& -g^{2} f^{a b e} f^{e c d}\left[D \phi^{a} \mid \eta\right]\left\langle\phi^{b} \mid \eta\right\rangle \frac{D}{-2 \partial^{+2}}\left[\phi^{c} \mid \eta\right]\left\langle D \phi^{d} \mid \eta\right\rangle
\end{aligned}
$$

where

$$
|\phi\rangle=\left[\begin{array}{c}
-\frac{\partial^{\wedge}}{\partial^{+}} \\
1
\end{array}\right] \phi ; \quad\left\langle\phi\left|=\left[\begin{array}{c}
1 \\
\frac{\partial^{\wedge}}{\partial^{+}}
\end{array}\right] \phi ; \quad\right| \bar{\phi}\right]=\left[\begin{array}{c}
1 \\
\frac{\partial^{\vee}}{\partial^{+}}
\end{array}\right] \bar{\phi} ; \quad\left[\bar{\phi} \mid=\left[\begin{array}{c}
-\frac{\partial^{\vee}}{\partial^{+}} \\
1
\end{array}\right] \bar{\phi}\right.
$$

The propagator can be read off

$$
\phi^{a} \bar{\phi}^{b} \sim \delta^{a b} \frac{2 \partial^{+}}{\partial^{2} D}
$$

or equivalently

$$
\left|\phi^{a}\right\rangle\left[\bar{\phi}^{b} \mid \sim \delta^{a b} \frac{2}{\partial^{2} D}\left[\begin{array}{cc}
\frac{\partial^{\vee} \partial^{\wedge}}{\partial^{+}} & -\partial^{\wedge} \\
-\partial^{\vee} & \partial^{+}
\end{array}\right]=\delta^{a b} \frac{2}{\partial^{2} D}\left(\frac{1}{\sqrt{2}} \not \partial-\frac{\partial^{2}}{2 \partial^{+}}|\eta\rangle[\eta \mid)\right.\right.
$$

The quartic term is written to look like two three-point vertices connected by a propagator. We observe that the effect of it is precisely to cancel the second term in the propagator. So we ignore the quartic term and use

$$
\left|\phi^{a}\right\rangle\left[\bar{\phi}^{b} \mid \sim \delta^{a b} \frac{\sqrt{2}}{\not \partial D}\right.
$$

as the propagator from now on. This observation is valid not only for the four point amplitudes, but for any Feynman diagrams.

\section{Some Intermediate Results}

\section{Self Mass Diagrams}

The upper entry applies to HV and the lower to DR schemes

$$
\begin{aligned}
\Pi(s, s) & =p^{2}\left\{\left[\begin{array}{l}
-8 \\
-8
\end{array}\right]+4\left[-\frac{1}{\epsilon}+\log \frac{p^{2} e^{\gamma}}{\mu^{2}}\right]+\text { IR terms }\right\} \\
\Pi(g-, g+; g) & =p^{2}\left\{\left[\begin{array}{l}
-67 / 9 \\
-64 / 9
\end{array}\right]+\frac{11}{3}\left[-\frac{1}{\epsilon}+\log \frac{p^{2} e^{\gamma}}{\mu^{2}}\right]+\text { IR terms }\right\} \\
\Pi(+,+; g) & =p^{+2}\left\{\left[\begin{array}{l}
5 / 9 \\
8 / 9
\end{array}\right]-\frac{1}{3}\left[-\frac{1}{\epsilon}+\log \frac{p^{2} e^{\gamma}}{\mu^{2}}\right]\right\}
\end{aligned}
$$




$$
\begin{aligned}
\Pi\left(g^{\mu}, g^{\nu} ; s\right) & =-\left(p^{2} g^{\mu \nu}-p^{\mu} p^{\nu}\right)\left\{\left[\begin{array}{c}
4 / 9 \\
4 / 9
\end{array}\right]-\frac{1}{6}\left[-\frac{1}{\epsilon}+\log \frac{p^{2} e^{\gamma}}{\mu^{2}}\right]\right\} \\
\Pi\left(g^{\mu}, g^{\nu} ; q\right) & =-\left(p^{2} g^{\mu \nu}-p^{\mu} p^{\nu}\right)\left\{\left[\begin{array}{c}
20 / 9 \\
20 / 9
\end{array}\right]-\frac{4}{3}\left[-\frac{1}{\epsilon}+\log \frac{p^{2} e^{\gamma}}{\mu^{2}}\right]\right\} \\
\Pi(q+, \bar{q}+) & =p^{2}\left\{\left[\begin{array}{l}
-7 \\
-6
\end{array}\right]+3\left[-\frac{1}{\epsilon}+\log \frac{p^{2} e^{\gamma}}{\mu^{2}}\right]+\text { IR terms }\right\}
\end{aligned}
$$

the IR terms for the self-mass are simple enough to be listed

$$
\operatorname{IR}_{\text {self-mass }}=\int_{0}^{p^{+}} d q^{+}\left[\frac{-2}{p^{+}-q^{+}}+\frac{-2}{q^{+}}\right]\left[-\frac{1}{\epsilon}+\log \frac{\left(p^{+}-q^{+}\right) q^{+} p^{2} e^{\gamma}}{\mu^{2} p^{+2}}\right]
$$

We observe that the quantity $\Pi\left(g^{I}, g^{J} ; g\right)+\Pi\left(g^{I}, g^{J} ; q\right) / 2-\Pi(q, \bar{q})$ is zero with $\delta$-regulator or DR, but non-zero with HV.

We also give the superficially divergent parts. To explain the notations, $D$ is the dimension in which tensor algebra or gamma matrix algebra is performed while $D_{1}$ is the dimension in which momentum integral is done. So by taking $D_{1}=D=2-2 \epsilon$ we obtain the HV result, and by taking $D=2, D_{1}=2-2 \epsilon$ we obtain the DR result. The indices $I, J \ldots$ range from 1 to 2 , and $i, j \ldots$ range from 1 to $2-2 \epsilon$. For HV we of course identify $I, J$ with $i, j$.

$$
\begin{aligned}
\Pi(s, s) & =p^{2} \frac{1}{\epsilon}(-4) \\
\Pi\left(g^{I}, g^{J} ; g\right) & =p^{2}\left[\frac{1}{\epsilon}\left(-4 \delta^{I J}+\frac{D}{3 D_{1}} \delta^{i j}\right)\right. \\
\Pi(+,+; g) & =p^{+2} \frac{1}{\epsilon} \frac{D}{6}+\text { Art. Div. } \\
\Pi\left(g^{I}, g^{J} ; s\right) & =p^{2} \delta^{i j} \frac{1}{\epsilon} \frac{1}{3 D_{1}} \\
\Pi(+,+; s) & =p^{+2} \frac{1}{\epsilon} \frac{1}{6} \\
\Pi\left(g^{I}, g^{J} ; q\right) & =p^{2} \frac{1}{\epsilon}\left(-\frac{4}{3 D_{1}} \delta^{i j}+2 \delta^{I J}\right) \\
\Pi(+,+; q) & =p^{+2} \frac{1}{\epsilon} \frac{4}{3} \\
\Pi(q, \bar{q}) & =-\frac{D}{2}\left[p_{\perp}^{i} \gamma^{i}-\frac{p_{\perp}^{2}}{2 p^{+}} \gamma^{+}-p^{+} \gamma^{-}\right]+\frac{(D-8) p^{2}}{4 p^{+}} \gamma^{+}+\text {Art. Div. }
\end{aligned}
$$

The results above all contain a factor of $i g^{2} N_{c} /\left(8 \pi^{2}\right) \operatorname{Tr}\left[t^{a} t^{b}\right]$.

\section{Triangle Diagrams}

We omit factor of $i g^{3} N_{c} /\left(8 \pi^{2}\right) \operatorname{Tr}\left[t^{a} t^{b} t^{c}\right]$

$$
\begin{aligned}
\Gamma(g-, g-, g+; s) & =\frac{-2 p_{3}^{+}}{p_{1}^{+} p_{2}^{+}} K_{2,1}^{\wedge}\left\{\left[\begin{array}{l}
-4 / 9 \\
-4 / 9
\end{array}\right]+\frac{1}{6}\left[-\frac{1}{\epsilon}+\log \frac{p_{o}^{2} e^{\gamma}}{\mu^{2}}\right]-\alpha \frac{1}{6} \frac{p_{1}^{+} p_{2}^{+}}{p_{3}^{+2}}\right\} \\
\Gamma(g-, g-, g+; q) & =\frac{-2 p_{3}^{+}}{p_{1}^{+} p_{2}^{+}} K_{2,1}^{\wedge}\left\{\left[\begin{array}{l}
-20 / 9 \\
-20 / 9
\end{array}\right]+\frac{4}{3}\left[-\frac{1}{\epsilon}+\log \frac{p_{o}^{2} e^{\gamma}}{\mu^{2}}\right]+\alpha \frac{2}{3} \frac{p_{1}^{+} p_{2}^{+}}{p_{3}^{+2}}\right\} \\
\Gamma(g-, g-, g+; g) & =\frac{-2 p_{3}^{+}}{p_{1}^{+} p_{2}^{+}} K_{2,1}^{\wedge}\left\{\left[\begin{array}{l}
67 / 9 \\
64 / 9
\end{array}\right]-\frac{11}{3}\left[-\frac{1}{\epsilon}+\log \frac{p_{o}^{2} \delta e^{\gamma}}{\mu^{2}}\right]-\alpha \frac{1}{3} \frac{p_{1}^{+} p_{2}^{+}}{p_{3}^{+2}}\right\}+\text { IR terms } \\
\Gamma(q+, \bar{q}+, g+) & =\frac{2 p_{2}^{+}}{q^{+} p_{1}} K_{p_{1}, q}^{\vee}\left\{\left[\begin{array}{l}
5 \\
6
\end{array}\right]-3\left[-\frac{1}{\epsilon}+\log \frac{p_{o}^{2} e^{\gamma}}{\mu^{2}}\right]\right\}+\text { IR terms }
\end{aligned}
$$




$$
\Gamma(s, s, g-)=\frac{2}{q^{+}} K_{2,1}^{\wedge}\left\{\left[\begin{array}{l}
8 \\
8
\end{array}\right]-4\left[-\frac{1}{\epsilon}+\log \frac{p_{o}^{2} e^{\gamma}}{\mu^{2}}\right]\right\}+\text { IR terms }
$$

where $\alpha=1$ if leg 3 is the off-shell leg $p_{o}$, and zero otherwise. It can be checked that the quantity $\Gamma(g, g, g ; g)+\Gamma(g, g, g ; q) / 2-\Gamma(q, \bar{q}, g)$ is zero only for $\delta$-regulator or DR.

Next, we give the superficially divergent parts. The external gluon self-mass diagrams have been included in this case to be able to get an artificial divergence free expression. We omit $-i g^{3} N_{c} /\left(8 \pi^{2}\right) \operatorname{Tr}\left[t^{a} t^{b} t^{c}\right]$

$$
\begin{aligned}
\Gamma\left(g^{I}, g^{J}, g^{K} ; g\right) & =\left[\frac{1}{\epsilon}\left(-\frac{1}{3}-\frac{D}{6}+\frac{13 D_{1}}{6}\right)+\frac{14}{3}\right] \delta^{I J} \frac{1}{p_{3}^{+}} K_{j i}^{k}+\text { cyc. perm } \\
\Gamma\left(g^{I}, g^{J}, g^{K} ; f\right) & =\left[\frac{1}{\epsilon}\left(6-D_{1}\right)-\frac{10}{3}\right] \delta^{I J} \frac{1}{p_{3}^{+}} K_{j i}^{k}+\frac{1}{\epsilon}\left(-\frac{8}{3}+\frac{2 D_{1}}{3}\right) \delta^{i j} \frac{1}{p_{3}^{+}} K_{j i}^{k}+\text { cyc. perm } \\
\Gamma\left(g^{I}, g^{J}, g^{K} ; s\right) & =-\left[\frac{1}{3} \delta^{I J} \frac{1}{p_{3}^{+}} K_{j i}^{k}+\frac{1}{\epsilon}\left(\frac{2}{3}-\frac{D_{1}}{6}\right) \delta^{i j} \frac{1}{p_{3}^{+}} K_{j i}^{k}+\text { cyc. perm }\right] \\
\Gamma\left(s, s, g^{K}\right) & =\left[\frac{1}{\epsilon}\left(-\frac{1}{3}-\frac{D}{6}+\frac{13 D_{1}}{6}\right)+5\right] \frac{1}{p_{3}^{+}} K_{j i}^{k} \\
\Gamma\left(q, \bar{q}, g^{K}\right) & =\left[\frac{1}{\epsilon}\left(\frac{1}{3}-\frac{D}{12}+\frac{5 D_{1}}{6}\right)+\frac{3}{2}\right] \gamma^{+} \frac{q^{i}}{q^{+}} \\
& +\left[\frac{1}{\epsilon}\left(-\frac{1}{2}-\frac{3 D_{1}}{4}\right)-\frac{3}{2}\right] \gamma^{K}+\frac{1}{\epsilon}\left(\frac{1}{6}+\frac{D-D_{1}}{12}\right) \gamma^{k} \\
& \left.+\left[\frac{1}{\epsilon}\left(-\frac{3}{4}+\frac{D}{8}+\frac{3 D_{1}}{8}\right)+\frac{3}{4}\right]\left(\gamma^{K} \gamma^{+} \frac{p / 1}{p_{1}^{+}}-\frac{p / 2}{p_{2}^{+}} \gamma^{K} \gamma^{+}\right)\right]
\end{aligned}
$$

In this list, only the terms containing $\not p / 1$ or $\not p / 2$ in $\Gamma\left(q, \bar{q}, g^{K}\right)$ have artificial divergence.

\section{References}

[1] M. B. Green, J. H. Schwarz and L. Brink, Nucl. Phys. B198 (1982) 474-492

Z. Bern and D. A. Kosower, Phys. Rev. Lett. 66:1669 (1991); Nucl. Phys. B379:451 (1992)

Z. Bern, L. Dixon and D. A. Kosower, Phys.Rev.Lett. 70 (1993) 2677-2680

[2] Z. Bern, L. J. Dixon and D. A. Kosower, Nucl. Phys. Proc. Suppl.51, 243(1996);

Annu. Rev. Nucl. Part. Sci. 46, 109(1996); J. High Energy Phys. 08(2004) 012

[3] F. Cachazo, P. Svrcek and E. Witten, J.High Energy Phys 09 (2004) 006; 010 (2004) 074; 010 (2004) 077

A. Brandhuber, B. Spence and G. Travaglini, Nucl. Phys. B 706 (2005) 150, hep-th/0407214.

[4] Z. Bern, M. Czakon, L. J. Dixon, D. A. Kosower and V. A. Smirnov, Phys. Rev. D75 (2007) 085010

Z. Bern, J. J. M.Carrasco, H. Johansson and D. A. Kosower, [arXiv:hep-th/0705.1864]

[5] Z. Bern, L. J. Dixon and V. A. Smirnov, Phys. Rev. D72, 085001 (2005) [arXiv:hep-th/0505205]

[6] Luis F. Alday and Juan Maldacena, J. High Energy Phys 06(2007) 064;

J. High Energy Phys 11(2007)068

[7] L. J. Dixon, [arXiv:hep-th/0803.2475]

[8] D. Chakrabarti, J. Qiu and C. B. Thorn, Phys. Rev. D72, 065022 (2005) [arXiv:hep-th/0507280].

[9] A. Gorsky and A. Rosly, J. High Energy Phys. 01(2006) 101

P. Mansfield, J. High Energy Phys 03(2006) 037

J. H. Ettle and T. R. Morris, J. High Energy Phys 08(2006) 003 
[10] A. Brandhuber, B. Spence, G. Travaglini and K. Zoubos

J. High Energy Phys. 07(2007) 002 [arXiv:hep-th/0704.0245]

[11] D. Chakrabarti, J. Qiu and C. B. Thorn, Phys. Rev. D74, 045018 (2006) [arXiv:hep-th/0602026].

[12] J. Qiu, Phys. Rev. D74, 085022(2006) [arXiv:hep-th/0607097].

[13] J. Qiu, Renormalization in the Light Cone World Sheet Formalism, Ph.D dissertation Univ. of Florida 08.2007

[14] G.'t Hooft and M. Veltman, Nucl. Phys. B44 (1972) 189

[15] W. Siegel, Phys. Lett. 84B 2 (1979)

[16] D. M. Capper, D. R. T. Jones and P. Van Nieuwenhuizen, Nucl. Phys. B167 (1980) 479-499

[17] L. Brink, J. H. Schwarz and J. Scherk, Nucl. Phys. B121 (1977) 71-92

[18] M.T. Grisaru and H.N.Pendleton, Nucl. Phys. B124 (1977) 81-92

[19] C. B. Thorn hep-th/0507213.

[20] Z. Kunszt, A. Signer and T. Trocsanyi Nucl.Phys B 411 (1994) 397 [arXiv:hep-ph/9305239].

[21] S. Mandelstam, Nucl. Phys. B213 (1983) 149

[22] A. Sen, Phys. Rev. D28 (1983) 860

N. Kidonakis, G. Oderda and G. Sterman, Nucl. Phys. B525 (1998) 299; B531 (1998) 365

[23] G. Sterman and M. E. Tejeda-Yeomans, Phys. Lett. B552, 48 (2003)

[24] L. Magnea and G. Sterman, Phys. Rev. D42, 4222 (1990) 